egneuro,02(02):64-65,2020

\title{
Choroidal Fissure Cyst Presenting as Hemi-Orofacial Sensory Loss
}

\author{
Karuna Tamrakar Karki ${ }^{1}$, Govinda Kandel ${ }^{2}$ \\ ${ }^{1}$ Department of Neurosurgery, B and C Medical College Teaching Hospital and Research Center, Birtamode, \\ Jhapa, Nepal. \\ ${ }^{2}$ Department of Internal Medicine, Mechi Zonal Hospital, Bhadrapur, Jhapa, Nepal.
}

\author{
Correspondence: \\ Dr. Karuna Tamrakar Karki \\ Department of Neurosurgery \\ B and C Medical College Teaching Hospital and Research Center, Birtamode, Jhapa, Nepal \\ Phone: +9779843405124 \\ Email: tamrakarkaruna@gmail.com
}

\begin{abstract}
Background: Choroidal fissure cysts are benign, asymptomatic, and discovered incidentally. CSF containing cysts at the level of the choroidal fissure are usually present with vague symptoms like a complex type of headache, cognitive disorder, tremor, paraesthesia, and visual disturbance. Here is a case report of a 33-yearold non-diabetic, non-smoker gentleman who had an insidious onset of loss of sensation over the left half of the face for the last 3 years which was secondary to a small choroidal fissure cyst diagnosed in MRI brain.
\end{abstract}

Ocs horoidal fissure cysts are benign, asymptomatic, and discovered incidentally. CSF containing cysts at the level of the choroidal fissure are usually present with vague symptoms like a complex type of headache, cognitive disorder, tremor, paraesthesia, and visual disturbance. ${ }^{1,2}$ Signal characteristics in MR images are identical to arachnid cyst. Although hemorrhagic transformation or rupture of an aneurysm into the cyst has been reported but the progression of such cysts is quite low. ${ }^{3}$

\section{Clinical features:}

A 33-year-old non-diabetic, non-smoker gentleman had an insidious onset of loss of sensation over the left half of the face for the last 3 years. He was unable to feel the movement of teeth and jawbones while chewing foods, which had been affecting his feeding. He experienced frequent food collection on the left side of the oral cavity while chewing food. More often he went unaware of forced biting leading to bite wounds in his tongue and mouth. Neurological examination revealed a sensory loss in V1, V2, and V3 division of the left trigeminal

64

Date submitted: $11 / 03 / 2020$

Date accepted: 09/05/2020 nerve. Jaw reflex was elicited. Other cranial nerves examination was normal. MRI showed CSF filled cyst in the medial temporal region, measuring about $10 \times 8 \mathrm{~mm} 2$ at the beginning of ambient cistern (Figure1).

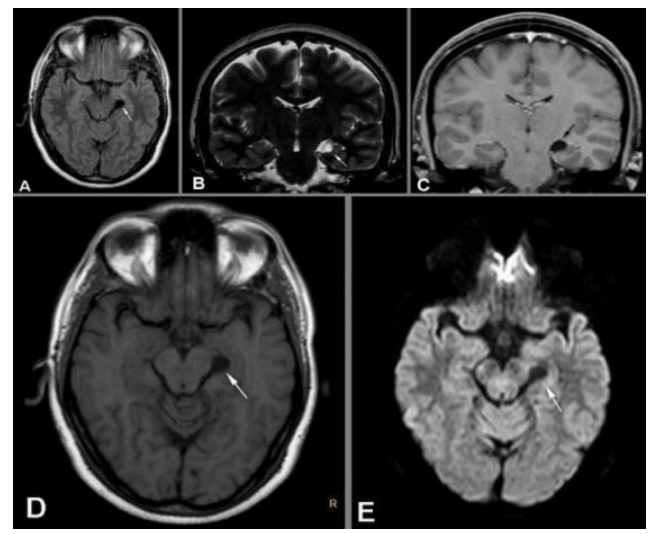

Figure 1: MRI of the patient. A: TI-hypointense, B: T2-hyperintense, C: no enhancement, D: FLAIRCSF intensity, E: DWI-diffusion restriction

It was hypointense in TI and hyperintense in T2 weighted images. There was neither perilesional edema nor contrast enhancement. FLAIR image egneuro, Volume 02, Issue 02, 2020 
followed CSF intensity, and signal intensity in DWI explained distinct diffusion restriction with a low coefficient map confirmed as an arachnoid cyst.

\section{Discussion:}

Mesencephalic nucleus, one of four trigeminal nerve nuclei, is a narrow crescent-shaped cellular organization situated in the lateral part of central gray matter around cerebral aqueduct and mesencephalic reticular formation at the mesopontine junction. ${ }^{4}$ These neurons represent an unusual diversity of primary sensory neurons. It is involved with the reflex proprioception of the periodontium and muscles of mastication. Its function is to prevent biting down hard enough to lose a tooth. Cell bodies located in the mesencephalic nucleus of the trigeminal nerve are actually connected to primary afferents primarily from muscle for chewing. Proprioceptive impulses are carried by fibers of pseudo-unipolar cells of mesencephalic nucleus bypassing the trigeminal ganglion. In fact, this is the only place in CNS where the cell bodies of primary afferent fibers are found within the CNS rather than in ganglia outside. Hence the mesencephalic nucleus is considered functionally as a sensory ganglion embedded within the brain stem. ${ }^{5}$ Unlike many nuclei within CNS, the mesencephalic nucleus contains no chemical synapses but is electrically coupled. Here, neurons are unipolar and receive proprioceptive information from the mandible and send projections to the trigeminal motor nucleus to mediate monosynaptic jaw reflexes. ${ }^{6}$

Choroid fissure is a narrow cleave between fornix and thalamus along which the choroid plexus is attached. Choroid fissure is developed at around 8 weeks of the embryonic period, when vascular pia mater invaginates into the medial wall of the cerebral hemisphere. ${ }^{3}$ Developmental anomalies may occur at the time of formation of primitive choroid plexus anywhere along the choroid fissure thus forming the cystic structure. ${ }^{2}$ Focal temporal hypoplasia resulting into dilatation of choroidal fissure may sometime mimic choroidal fissure cyst formation. These types of cyst may be of a neuroepithelial or arachnoid cyst. They are usually small round to oval in shape measuring between 1$2 \mathrm{~cm}^{2}$ in size. Choroidal fissure cyst associated with complex partial seizure has been reported by
Morioka $\mathrm{T}$ et al. ${ }^{7}$ De Jong et al. reported 6 cases of CSF containing choroidal fissure cyst presented with headache, narcoplexy, and hyperactivity disorder. ${ }^{1}$ MR Studies of CSF like Choroidal fissure cyst in 26 cases done by Sherman et al. had presenting symptoms like complex migraine, seizure, gait disturbance, hemiparesis, tremor, vertigo, hearing loss and visual scotomas. ${ }^{2}$ Most of them were adults and though one had presented with paraesthesia, however hemisensory facial numbness and loss of periodontium proprioception has not been reported yet in the English Literature. The trigeminal nerve supplies meninges and may produce a complex type of headache. The involvement of facial sensory pathways with mesencephalic features i.e. loss of sensation from the periodontium is extremely rare. The medial temporal lobe being an epileptogenic choroidal fissure cyst may produce complex partial seizure or temporal lobe seizure due to irritation of the trigeminal fibers rather than a compressive effect.

\section{Conclusion:}

Choroidal fissure cysts though are small and incidentally picturized in MRI, they may sometimes present with exclusive symptoms of Hemi orofacial sensory loss.

\section{References:}

1. C de Jong L, Thewissen L, Van Loon J, Van Calenbergh F. Choroidal fissure cerebrospinal fluid-containing cysts: case series, anatomical consideration, and review of the literature. World Neurosurg 2011; 75:704-708.

2. Sherman JL, Camponovo E, Citrin CM. MR imaging of CSF-like choroidal fissure and parenchymal cysts of the brain. AJR Am J Roentgenol 1990; 155:1069-1075.

3. Karatas A, Gelal F, Gurkan G, Feran H. Growing Hemorrhagic Choroidal Fissure Cyst. J Korean Neurosurg Soc 2016; 59:168-171.

4. Leston JM. Functional anatomy of the trigeminal nerve. Neurochirurgi 2009; 55:99-112.

5. Shigenaga Y, Doe K, Suemune S, Mitsuhiro Y, Tsuru K, Otani K, et al. Physiological and morphological characteristics of periodontal mesencephalic trigeminal neurons in the cat--intra-axonal staining with HRP. Brain Res 1989; 505:91-110.

6. Khalid S, Tubbs RS. Neuroanatomy and Neuropsychology of Pain. Cureus 2017;9: e1754.

7. Morioka T, Nishio S, Suzuki S, Fukui M, Nishiyama T. Choroidal fissure cyst in the temporal horn associated with complex partial seizure. Clin Neurol Neurosurg 1994; 96:164-167. 